\title{
Analytical derivation of a general 2D non-prismatic beam model based on the Hellinger-Reissner principle
}

\author{
Angela Beltempo ${ }^{\mathrm{a}}$, Giuseppe Balduzzi ${ }^{\mathrm{b}}$, Giulio Alfano ${ }^{\mathrm{c}}$, Ferdinando Auricchio ${ }^{\mathrm{b}}$ \\ ${ }^{a}$ Department of Civil, Environmental and Mechanical Engineering, Università degli Studi di Trento, Trento, Italy \\ ${ }^{b}$ Department of Civil Engineering and Architecture, Università degli Studi di Pavia, Pavia, Italy \\ ${ }^{c}$ Department of Mechanical, Aerospace and Civil Engineering, Brunel University, London, United Kingdom
}

\begin{abstract}
This paper presents an analytical model for the study of 2D linear-elastic non-prismatic beams. Its principal aim is to accurately predict both displacements and stresses using a simple procedure and few unknown variables. The approach adopted for the model derivation is the so-called dimensional reduction starting from the Hellinger-Reissner (HR) functional, which has both displacements and stresses as independent variables. Furthermore, the Timoshenko beam kinematic and appropriate hypotheses on the stress field are considered in order to enforce the boundary equilibrium. The use of dimensional reduction allows to reduce the integral over a 2D domain, associated with the HR functional, into an integral over a 1D domain (i.e., the so-called beam-axis). Finally, through some mathematical manipulations, the paper shows how to obtain the six Ordinary Differential Equations (ODEs) governing the beam structural behaviour. In order to show the proposed model capabilities, the paper illustrates the ODEs solutions for several non-prismatic beams with different geometries, constraints, and load distributions. The results are compared with the ones provided by an already existing, more expensive, and refined 2D finite element analysis, showing the efficiency of the proposed model to accurately predict both displacements and stresses, at least in cases of practical interest.
\end{abstract}

Keywords: non-prismatic beam, analytical beam model, dimensional reduction, mixed variational formulation, boundary equilibrium

\section{Introduction}

Non-prismatic beams are slender bodies in which the cross-section parameters (e.g., dimensions, centroid position, shape) could vary along the beam longitudinal axis. Practitioners are interested in this class of bodies since it is possible to optimize their shape according to the design requirements. As an example, if the cross-section size varies proportionally to the internal-stress magnitude, the beam achieves the required structural strength with the minimum amount of material. Therefore, non-prismatic elements are widely used in many engineering fields, such as the design of bridges, biomedical devices, and blades. Obviously, it is necessary to consider the effects of cross-section variation through adequately refined models, otherwise the benefits of geometry cannot be caught.

Nowadays, Finite Element (FE) analyses based on 3D body full discretization could provide extremely accurate descriptions. However, the use of 1D models, such as beams, still represents the most convenient choice for many applications, at least in civil engineering. Unfortunately, an effective non-prismatic beam modelling is still a nontrivial issue, as the following literature review demonstrates.

The simplest approach proposed in literature for non-prismatic beam modelling assumes the Euler-Bernoulli and Timoshenko beam stiffness-coefficients as functions of the beam-axis coordinate. Portland Cement Association [1]

${ }^{*}$ Corresponding author. Adress: Department of Civil, Environmental and Mechanical Engineering, Università degli Studi di Trento, via Mesiano 77, 38123 Trento Italy Email adress: angela. beltempo@unitn.it Phone: 00390461282547

Email addresses: angela.beltempo@unitn.it (Angela Beltempo), giuseppe.balduzzi@unipv.it (Giuseppe Balduzzi), Giulio.Alfano@brunel.ac.uk (Giulio Alfano), ferdinando.auricchio@unipv.it (Ferdinando Auricchio) 
uses this approach to evaluate the stiffness of several non-prismatic beams and it still represents a milestone for structural design, as the frequent references to the handbook demonstrate [2, 3, 4]. Banerjee and Williams [5] illustrate another example of the classical beam theory generalization to non-prismatic beams. Specifically, the authors evaluate the FE static stiffness matrix for a range of non-prismatic beam-columns, considering the cross-section area, the second moment of area, and the torsional rigidity as functions of the beam-axis coordinate. Similarly, Friedman and Kosmatka [6] propose a procedure to evaluate axial, bending, and torsional stiffness coefficients based on opportune modifications of prismatic beam theory coefficients. Colunga [2] and Ozay and Topcu [7] present similar approaches and apply them to a wide range of span load variations.

Unfortunately, for beams with a rapid cross-section variation, the error induced by the poorness of the so far mentioned procedures may grow significantly. Specifically, their main limitations are listed in the following.

- Simplified geometry. Only beams that are symmetric and with a linear cross-section variation along the beamaxis are generally considered.

- Negligible taper angle. Approaches based on the classical beam theories are not sufficiently accurate for studying beams with a significant cross-section variation, as well-known since the sixties [8,9]. The same applies for Jourawsky formula widely used to recover the shear stress distributions [10,11].

- Independence among axial, shear-bending, and torsional equations. The simplest approaches are also not able to capture the flexural and extensional coupling [2, 4, 12]. In particular, to overcome this problem, [2, 4] propose refined $3 \mathrm{D} \mathrm{FE}$ analysis in order to evaluate the real stiffness of non-prismatic elements.

Limiting the attention to stress analyses, Timoshenko and Goodier [13] provide the analytical expression of stress distributions for an infinite long wedge which consists of the combination of some trigonometric functions. It is worth nothing that, the same expression could be used, under suitable hypotheses, for non-prismatic beams with linear variation of the cross-section size [11]. Bleich [14] provides another method, known as effective shearing force, for evaluating the maximum shear stress within the cross-section. Later on, this method has been introduced in many technical codes $[15,16]$ and related documents, becoming the standard stress-analysis procedure for practitioners. The effective shear force formula is reported also in some classical books of continuum mechanics [17]. Unfortunately, despite its consolidated use, Paglietti and Carta $[18,19]$ highlight that this formula could be extremely inaccurate, leading to underestimated shear strength values.

Hodges et al. [20] propose a more refined model for planar non-prismatic beams based on the variational asymptotic method. The authors introduce an accurate model and investigate the effects of the lateral-surface slope associated with the taper angle. In particular, they prove that the slope is a crucial parameter for the boundary equilibrium definition. Hodges et al. [12] integrate the same model proposing an effective stress and strain recovery procedure. Unfortunately, they limit the study to symmetrically non-prismatic beams.

With regard to non-symmetrically non-prismatic beams, a model considering a curvilinear centreline and a constant cross-section depth (the so-called arch-beams) was introduced at the beginning of the past century [21]. However, to the authors' knowledge, a model capable of effectively describing the behaviour of generic non-prismatic beams is still not available in literature.

To overcome the limitations observed in previous studies, this paper proposes an advanced analytical, 2D, linearelastic, non-prismatic beam model, considering also complex situations such as non-linear and rapid cross-section variations, as well as symmetric and non-symmetric configurations. This work generalizes the procedure illustrated in Auricchio et al. [22], which uses dimensional reduction and the Hellinger-Reissner functional for the modelling of 2D linear-elastic prismatic beams. Balduzzi [23] presents a study of 2D linear-elastic non-prismatic beams following the same procedure and obtaining encouraging results. However, a high number of variables characterizes the beam model, which could undermine the method efficiency.

Therefore, one of the aims of the present work is to explore the accuracy achievable through a reduced number of variables. Moreover, in order to facilitate further developments, the model will satisfy the conditions listed below.

- It does not need correction factors, whose evaluation could interfere with the model effectiveness.

- It takes adequately into account boundary conditions, which results to be a crucial factor in non-prismatic beam-model development. 
- It has a clear and consistent derivation path.

The paper structure is detailed in the following: Section 2 defines the considered 2D problem, introduces the variational formulation, and provides the displacement and stress field hypotheses; Section 3 develops the analytical beam model and derives the ODEs, which govern the behaviour of the non-prismatic beam under investigation; Section 4 presents several examples of non-prismatic beam and compares the results obtained through the proposed analytical model with the ones provided by other methods (both analytical and numerical). Finally, Section 5 resumes the model capabilities and discusses further developments.

\section{Problem formulation}

This section introduces the 2D problem to be tackled and all the assumptions necessary for the model formulation. Specifically, Subsection 2.1 introduces the problem domain, Subsection 2.2 presents the HR variational formula to be used as starting equation, and Subsection 2.3 illustrates the hypotheses to be considered within the modelling derivation procedure.

\subsection{Geometry definition}

The object under investigation is a planar beam with a generic non-prismatic shape and described as a 2D domain $\Omega$. In particular, we refer to a case in which the plane-stress-state hypothesis can be imposed to a 3D body since its width is negligible, and we limit to the case of small displacements, small strains, and linear-elastic isotropic material.

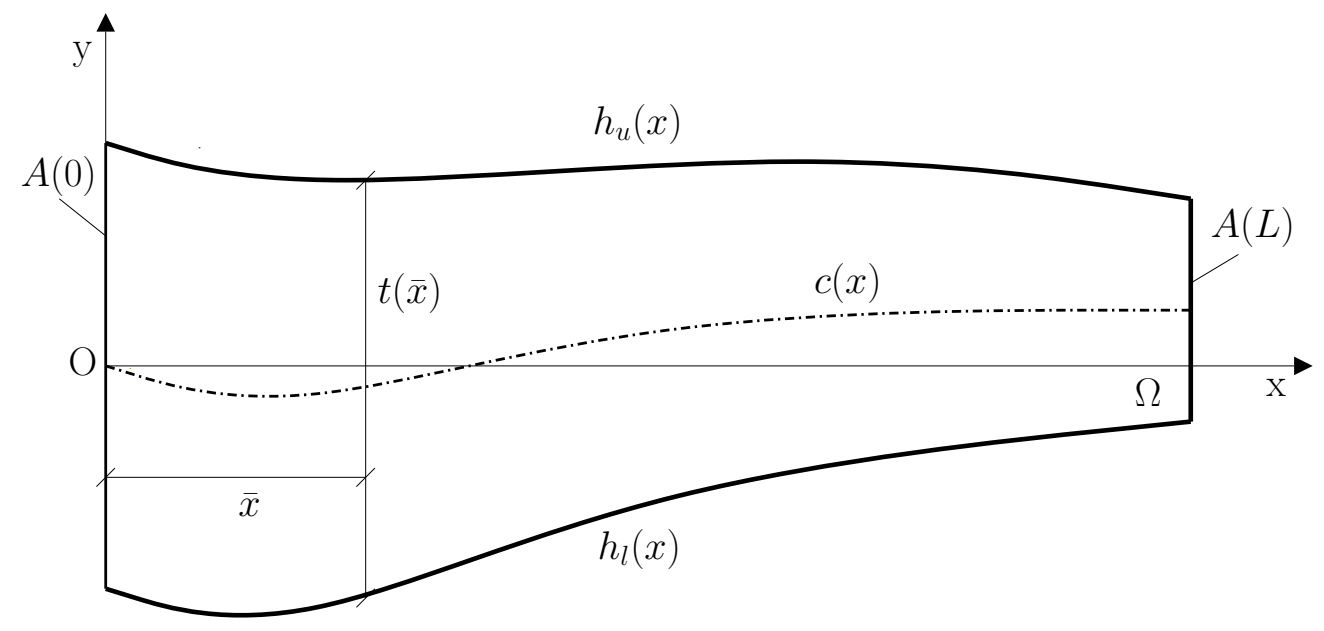

Figure 1: Generic non-prismatic beam

As shown in Figure 1, the $x$ axis is a suitably chosen straight and normal line to the cross-sections and the $y$ axis has the direction of the (variable) cross-section depth. In order to define the domain $\Omega$, we introduce two sufficiently smooth functions $h_{u}(x)$ and $h_{l}(x)$ (such that $h_{l}(x)<h_{u}(x) \forall x \in \ell$ ) able of describing the geometry of the upper and lower limits of the domain. As a consequence, $\Omega$ can be rigorously defined in relation to the beam longitudinal axis $\ell$ (a closed and bounded subset of the $x$ Cartesian-coordinate axis) and the cross-section $A(x)$ (a closed and bounded subset of the $y$ Cartesian-coordinate axis). As usual for beams, the longitudinal axis $\ell$ is assumed to be the predominant dimension of the body. Then, the dependence on $x$ of the subset $A(x)$ is emphasized on indicating the variation of the cross-section area values along the beam-axis. In summary, the domain $\Omega$ is defined as follows:

$$
\Omega \subset \mathbb{R}^{2} \mid x \in \ell \text { and } y \in A(x)
$$

where

$$
\ell=\{x \in \mathbb{R} \mid x \in[0, L]\}, A(x)=\left\{y \in \mathbb{R} \mid y \in\left[h_{l}(x), h_{u}(x)\right]\right\}
$$


At this point, the domain boundary, $\partial \Omega=A(0) \cup A(L) \cup h_{u}(x) \cup h_{l}(x)$ (see Figure 1), can be divided into two parts $\partial \Omega_{t}$ and $\partial \Omega_{s}$, where load and displacement constrains are imposed respectively.

With regard to the definition of $h_{u}(x)$ and $h_{l}(x)$, we find convenient to introduce two new functions: the centreline $c(x)$, which defines the $y$ coordinates of the cross-section centroids, and the thickness $t(x)$, which is the cross-section depth assumed as a positive definite function. Therefore, $h_{u}(x)$ and $h_{l}(x)$ can be expressed as function of $c(x)$ and $t(x)$ :

$$
\begin{aligned}
& h_{u}(x)=c(x)+\frac{t(x)}{2} \\
& h_{l}(x)=c(x)-\frac{t(x)}{2}
\end{aligned}
$$

\subsection{Variational formulation}

In what follows, the symmetric stress tensor $\sigma$ and the displacement vector $\boldsymbol{s}$ are the independent variable fields, $\boldsymbol{D}$ is the inverse of the fourth order elastic tensor, $\boldsymbol{f}$ is the distributed load vector, $\boldsymbol{n}$ indicates the outward unit vector defined on $\partial \Omega, \bar{s}$ is the sufficiently-smooth boundary displacement assigned on $\partial \Omega_{s}$, and $t$ is the boundary load distribution assigned on $\partial \Omega_{t}$.

The functional spaces $W, S_{0}$, and $S_{t}$ are defined as follows:

$$
\begin{aligned}
W & :=\left\{\boldsymbol{s} \in L^{2}(\Omega)\right\} \\
S_{0} & :=\left\{\boldsymbol{\sigma} \in H(\operatorname{div}, \Omega):\left.\boldsymbol{\sigma} \cdot \boldsymbol{n}\right|_{\partial \Omega_{t}}=\mathbf{0}\right\} \\
S_{t} & :=\left\{\boldsymbol{\sigma} \in H(\operatorname{div}, \Omega):\left.\boldsymbol{\sigma} \cdot \boldsymbol{n}\right|_{\partial \Omega_{t}}=\boldsymbol{t}\right\}
\end{aligned}
$$

where the Sobolev spaces $L^{2}(\Omega)$ and $H(\operatorname{div}, \Omega)$ are explicit hereunder:

$$
\begin{aligned}
L^{2}(\Omega) & :=\left\{s: \Omega \rightarrow \mathbb{R}^{2}: \int_{\Omega} s \cdot s d \Omega<\infty\right\} \\
H(\operatorname{div}, \Omega) & :=\left\{\sigma: \Omega \rightarrow \mathbb{R}_{s}^{2 \times 2}: \int_{\Omega} \sigma: \sigma d \Omega<\infty \text { and }(\nabla \cdot \sigma) \in L^{2}(\Omega)\right\}
\end{aligned}
$$

Dimensional reduction starts from the HR principle which characterizes the problem solution as the unique saddle point of the following functional:

$$
\begin{array}{r}
J_{H R}(\boldsymbol{s}, \boldsymbol{\sigma})=\int_{\Omega}(\nabla \cdot \boldsymbol{\sigma} \cdot \boldsymbol{s}) d \Omega-\frac{1}{2} \int_{\Omega}(\boldsymbol{\sigma}: \boldsymbol{D}: \boldsymbol{\sigma}) d \Omega-\int_{\Omega}(\boldsymbol{s} \cdot \boldsymbol{f}) d \Omega \\
-\int_{\partial \Omega_{s}}(\boldsymbol{\sigma} \cdot \boldsymbol{n} \cdot \overline{\boldsymbol{s}}) d S=0
\end{array}
$$

It is worth noticing that there are several formulations of HR functional [24, 25, 22]. Specifically, we adopt the formulation indicated with $H R d i v$-div by Auricchio et al. [22], in which it was successfully applied to multilayered planar beams.

Computing the stationary point of the HR functional (7), the following variational formula is obtained:

$$
\begin{aligned}
\text { Find } \boldsymbol{s} & \in W \text { and } \boldsymbol{\sigma} \in S_{t} \text { such that } \forall \delta \boldsymbol{s} \in W \text { and } \forall \delta \boldsymbol{\sigma} \in S_{0} \\
\delta J_{H}= & -\int_{\Omega} \delta \boldsymbol{s} \cdot \nabla \cdot \boldsymbol{\sigma} d \Omega-\int_{\Omega} \nabla \cdot \delta \boldsymbol{\sigma} \cdot \boldsymbol{s} d \Omega-\int_{\Omega} \delta \boldsymbol{\sigma}: \boldsymbol{D}^{-1}: \boldsymbol{\sigma} d \Omega \\
& -\int_{\Omega} \delta \boldsymbol{s} \cdot \boldsymbol{f} d \Omega+\int_{\partial \Omega_{s}} \delta \boldsymbol{\sigma} \cdot \boldsymbol{n} \cdot \overline{\boldsymbol{s}} d S=0
\end{aligned}
$$

In Equation (8) the displacement constrain $\left.s\right|_{\partial \Omega_{s}}=\bar{s}$ is the natural boundary condition, whereas the boundary equilibrium $\left.\boldsymbol{\sigma} \cdot \boldsymbol{n}\right|_{\partial \Omega_{t}}=\boldsymbol{t}$ is the essential boundary condition directly enforced on $S_{t}$ (see Equation (6)). Alessandrini et al. 
[26] use the HR functional formulation (8) for the derivation of some plate models, providing mathematical proof of modelling error. Moreover, HR functional (8) is different from the second statement of HR functional proposed by Bauchau and Craig [24] because of the omission of the integral over the loaded boundary.

Since Equation (8) represents the saddle point of the HR mixed functional, it requires an accurate selection of both displacement and stress functions in order to ensure the problem is well-posed [27]. This aspect could lead to difficulties in the formulation because of the complexity of field variables $[28,29]$. However, the HR functional use gives great advantages in terms of the stress distribution accuracy, especially if compared with the one-field functionals, such as the Total Potential Energy [22].

\subsection{Hypotheses on displacement and stress fields}

In order to apply dimensional reduction, we need to introduce suitable hypotheses on the unknown fields, $s$ and $\sigma$, expressed in the following as functions of their scalar components:

$$
\boldsymbol{s}=\left(\begin{array}{l}
u_{s}(x, y) \\
v_{s}(x, y)
\end{array}\right) \quad \boldsymbol{\sigma}=\left[\begin{array}{ll}
\sigma_{x x}(x, y) & \sigma_{x y}(x, y) \\
\sigma_{x y}(x, y) & \sigma_{y y}(x, y)
\end{array}\right]
$$

The procedure starts assuming the horizontal $u_{s}(x, y)$ and the vertical $v_{s}(x, y)$ displacement components to be respectively a linear and a constant function of $y$, where $u(x), \theta(x)$ and $v(x)$ are the new displacement independent variables of the problem.

$$
\begin{aligned}
& u_{s}(x, y)=u(x)+\tilde{y} \frac{t(x)}{2} \theta(x) \\
& v_{s}(x, y)=v(x)
\end{aligned}
$$

More precisely, $u(x)$ indicates the displacement in $x$ direction, $\theta(x)$ represents the rigid rotation of the cross-section with respect to the undeformed configuration, and $v(x)$ is the displacement in $y$ direction. Finally, $\tilde{y}$ is a linear function of $y$, defined in order to be equal to 1 at $h_{u}(x), 0$ at $c(x)$, and -1 at $h_{l}(x)$ :

$$
\tilde{y}=(-c(x)+y) \frac{2}{t(x)}
$$

At this point, it can be noticed that the so far introduced displacement variables, $u_{s}(x, y)$ and $v_{s}(x, y)$, correspond to the displacement variables of Timoshenko prismatic beam theory.

Before introducing the hypotheses on the stress tensor components, it is important to discuss the essential condition of the HR variational formula to be imposed on the upper and lower limits of the domain:

$$
\left.(\boldsymbol{\sigma} \cdot \boldsymbol{n})\right|_{h_{u}}=\left.\mathbf{0} \quad(\boldsymbol{\sigma} \cdot \boldsymbol{n})\right|_{h_{l}}=\mathbf{0}
$$

The analytical expressions of $\left.\boldsymbol{n}\right|_{h_{u}}$ and $\left.\boldsymbol{n}\right|_{h_{l}}$ are reported in the following:

$$
\left.\mathbf{n}\right|_{h_{u}}=\left.\frac{1}{\sqrt{1+h_{u}^{\prime}(x)^{2}}}\left(\begin{array}{c}
-h_{u}^{\prime}(x) \\
1
\end{array}\right) \quad \mathbf{n}\right|_{h_{l}}=\frac{1}{\sqrt{1+h_{l}^{\prime}(x)^{2}}}\left(\begin{array}{c}
h_{l}^{\prime}(x) \\
-1
\end{array}\right)
$$

Introducing Definitions (13) in Equations (12), all the stress components can be expressed in terms of the horizontal stress $\sigma_{x x}(x, y)$, which becomes the unique stress variable necessary to define the stress state on the lateral surface:

$$
\left.\left[\begin{array}{ll}
\sigma_{x x}(x, y) & \sigma_{x y}(x, y) \\
\sigma_{x y}(x, y) & \sigma_{y y}(x, y)
\end{array}\right] \cdot\left(\begin{array}{l}
n_{x} \\
n_{y}
\end{array}\right)\right|_{h_{u / l}}=\left(\begin{array}{l}
0 \\
0
\end{array}\right) \rightarrow\left\{\begin{array}{c}
\left.\sigma_{x y}(x, y)\right|_{h_{u / l}}=-\left.\left(n_{x} / n_{y}\right) \sigma_{x x}(x, y)\right|_{h_{u / l}} \\
\left.\sigma_{y y}(x, y)\right|_{h_{u / l}}=\left.\left(n_{x} / n_{y}\right)^{2} \sigma_{x x}(x, y)\right|_{h_{u / l}}
\end{array}\right.
$$

For the upper limit the following expressions are obtained:

$$
\left.\sigma_{x y}(x, y)\right|_{h_{u}}=\left.h_{u}^{\prime}(x) \sigma_{x x}(x, y)\right|_{h_{u}}
$$




$$
\left.\sigma_{y y}(x, y)\right|_{h_{u}}=\left.h_{u}^{\prime}(x)^{2} \sigma_{x x}(x, y)\right|_{h_{u}}
$$

and for the lower limit the following expressions are obtained:

$$
\begin{aligned}
& \left.\sigma_{x y}(x, y)\right|_{h_{l}}=\left.h_{l}^{\prime}(x) \sigma_{x x}(x, y)\right|_{h_{l}} \\
& \left.\sigma_{y y}(x, y)\right|_{h_{l}}=\left.h_{l}^{\prime}(x)^{2} \sigma_{x x}(x, y)\right|_{h_{l}}
\end{aligned}
$$

We assume that $\sigma_{x x}(x, y)$ and $\sigma_{y y}(x, y)$ are linear functions of $y$, whereas $\sigma_{x y}(x, y)$ is a quadratic function of $y$, as detailed hereunder:

$$
\begin{aligned}
& \sigma_{x x}(x, y)=\sigma_{x 0}(x)+\tilde{y} \sigma_{x 1}(x) \\
& \sigma_{y y}(x, y)=\left.\sigma_{y y}(x, y)\right|_{h_{l}} \frac{h_{u}(x)-y}{h_{u}(x)-h_{l}(x)}+\left.\sigma_{y y}(x, y)\right|_{h_{u}} \frac{y-h_{l}(x)}{h_{u}(x)-h_{l}(x)} \\
& \sigma_{x y}(x, y)=\left.\sigma_{x y}(x, y)\right|_{h_{l}} \frac{h_{u}(x)-y}{h_{u}(x)-h_{l}(x)}+\left.\sigma_{x y}(x, y)\right|_{h_{u}} \frac{y-h_{l}(x)}{h_{u}(x)-h_{l}(x)}+\tilde{b} \tau(x)
\end{aligned}
$$

where $\tilde{b}$ represents the so-called bubble function, i.e. a quadratic function equal to 0 at $h_{u}(x)$ and $h_{l}(x)$, and -1 at $c(x)$. The function $\tilde{b}$ is analytically defined as:

$$
\tilde{b}=(-c(x)+y)^{2} \frac{4}{t(x)^{2}}-1
$$

Substituting Equations (15) and (16) in Equation (17), the stress tensor components can be written as functions of $\sigma_{x 0}(x), \sigma_{x 1}(x)$ and $\tau(x)$, which are the new stress independent variables.

$$
\begin{aligned}
\sigma_{x x}(x, y)= & \sigma_{x 0}(x)+\tilde{y} \sigma_{x 1}(x) \\
\sigma_{y y}(x, y)= & h_{l}^{\prime}(x)^{2}\left[\sigma_{x 0}(x)-\sigma_{x 1}(x)\right] \frac{h_{u}(x)-y}{h_{u}(x)-h_{l}(x)}+h_{u}^{\prime}(x)^{2}\left[\sigma_{x 0}(x)+\sigma_{x 1}(x)\right] \frac{y-h_{l}(x)}{h_{u}(x)-h_{l}(x)} \\
\sigma_{x y}(x, y)= & h_{l}^{\prime}(x)\left[\sigma_{x 0}(x)-\sigma_{x 1}(x)\right] \frac{h_{u}(x)-y}{h_{u}(x)-h_{l}(x)}+h_{u}^{\prime}(x)\left[\sigma_{x 0}(x)+\sigma_{x 1}(x)\right] \frac{y-h_{l}(x)}{h_{u}(x)-h_{l}(x)}+ \\
& +\tilde{b} \tau(x)
\end{aligned}
$$

More precisely, $\sigma_{x 0}(x)$ represents the axial stress contribution, $\sigma_{x 1}(x)$ is related to the bending stress, and $\tau(x)$ is the shear stress contribution.

Concluding, we have introduced six independent variables: three displacements and three stresses, which correspond to the usual variables adopted in most of the first order beam theories. Furthermore, comparing the assumptions on stress distributions so far introduced with the analytical solution of an infinite long wedge [13], they result adequate in describing the analytical solution for relatively small lateral surface slope. In fact, Timoshenko and Goodier [13] notice that, for a axially loaded wedge, the ratio between minimum and maximum axial stress is proportional to $\cos ^{4}(\alpha)$ being $\alpha$ the slope of the wedge edges.

\section{Analytical derivation of the differential equations}

This section illustrates the procedure to derive the ODEs governing the beam behaviour. Henceforward, in order to simplify the equation reading, the dependence on $x$ after each variable is omitted.

Assuming $\overline{\boldsymbol{s}}=\mathbf{0}$ and $\boldsymbol{f} \neq \mathbf{0}$ (whose two components are generically indicated with $f_{1}$ and $f_{2}$ ), the stationarity of the HR functional reduces to the following expression:

$$
\delta J_{H R}(\boldsymbol{s}, \boldsymbol{\sigma})=-\int_{\Omega}(\delta \boldsymbol{s} \cdot \nabla \cdot \boldsymbol{\sigma}) d \Omega-\int_{\Omega}(\nabla \cdot \delta \boldsymbol{\sigma} \cdot \boldsymbol{s}) d \Omega-\int_{\Omega}(\delta \boldsymbol{\sigma}: \boldsymbol{D}: \boldsymbol{\sigma}) d \Omega-\int_{\Omega}(\delta \boldsymbol{s} \cdot \boldsymbol{f}) d \Omega=0
$$


Introducing the displacement and stress approximations (10) and (19), the integral over $\Omega$ is split into two integrals over the two subsets $\ell$ and $A(x)$. Analytically solving the integrals over $A(x)$, Equation (8) reduces to the following integral over $\ell$ :

$$
\begin{aligned}
\delta J_{H R}(\boldsymbol{s}, \boldsymbol{\sigma})=- & \int_{0}^{L}\left[\delta u M+\delta \nu O+\delta \theta Q+\delta \sigma_{\mathrm{x} 0} R+\delta \sigma S_{\mathrm{x} 1}\right] d x \\
& -\int_{0}^{L}\left[\delta \tau T+\delta \sigma_{\mathrm{x} 0}^{\prime} U+\delta \sigma_{\mathrm{x} 1}^{\prime} V+\delta \tau^{\prime} Z\right] d x=0
\end{aligned}
$$

where the coefficients $M, O, Q, R, S, T, U, V, Z$ result defined as follows:

$$
\begin{aligned}
M= & t^{\prime} \sigma_{x 0}+t \sigma_{x 0}^{\prime}+t f_{1} \\
O= & 6\left(t^{\prime} c^{\prime}+t c^{\prime \prime}\right) \sigma_{x 0}+6 t c^{\prime} \sigma_{x 0}^{\prime}+3\left(t^{\prime 2}+t t^{\prime \prime}\right) \sigma_{x 1}+3 t t^{\prime} \sigma_{x 1}^{\prime}-4 t^{\prime} \tau-4 t \tau^{\prime}+6 t f_{2} \\
Q= & -t^{\prime} \sigma_{x 1}+t \sigma_{x 1}^{\prime}+4 \tau \\
R= & t^{\prime} u+\left(t^{\prime} c^{\prime}+t c^{\prime \prime}\right) v+\frac{t}{48 E}\left[48+48 c^{\prime 4}+(8-16 v) t^{\prime 2}+3 t^{\prime 4}+96 c^{\prime 2}+40 t^{\prime 2} c^{\prime 2}\right] \sigma_{x 0}+ \\
& \frac{t t^{\prime} c^{\prime}}{3 E}\left(4+4 c^{\prime 2}+t^{\prime 2}\right) \sigma_{x 1}-\frac{4 t}{3 E}(1+v) c^{\prime} \tau \\
S= & \frac{\left(t^{\prime 2}+t t^{\prime \prime}\right)}{2} v-\frac{t t^{\prime}}{6} \theta+\frac{t t^{\prime} c^{\prime}}{3 E}\left(4+4 c^{\prime 2}+t^{\prime 2}\right) \sigma_{x 0}-\frac{2 t t^{\prime}}{3 E}(1+v) \tau+ \\
& \frac{t}{48 E}\left[16+16 c^{\prime 4}+8(3+2 v) t^{\prime 2}+t^{\prime 4}+32 c^{\prime 2}+56 t^{\prime 2} c^{\prime 2}\right] \sigma_{x 1} \\
T= & -\frac{2 t^{\prime}}{3} v+\frac{2 t}{3} \theta-\frac{4 t c^{\prime}}{3 E}(1+v) \sigma_{x 0}-\frac{2 t t^{\prime}}{3 E}(1+v) \sigma_{x 1}+\frac{16 t}{15 E}(1+v) \tau \\
U= & t u+t c^{\prime} v \\
V= & \frac{1}{6}\left(3 t t^{\prime} v+t^{2} \theta\right) \\
Z= & -\frac{2}{3} t v \\
&
\end{aligned}
$$

Integrating by parts the terms $\delta \sigma_{x 0}^{\prime} U, \delta \sigma_{x 1}^{\prime} V$ and $\delta \tau^{\prime} Z$, the variational formula is obtained:

$$
\begin{array}{r}
\delta J_{H R}(\boldsymbol{s}, \boldsymbol{\sigma})=-\int_{0}^{L}\left[\delta u M+\delta v O+\delta \theta Q+\delta \sigma_{\mathrm{x} 0}\left(R-U^{\prime}\right)+\delta \sigma_{\mathrm{x} 1}\left(S-V^{\prime}\right)\right] d x \\
-\int_{0}^{L}\left[\delta \tau\left(T-Z^{\prime}\right)\right] d x+[\cdot \cdot]_{0}^{L}=0
\end{array}
$$

where $[\cdot \cdot \cdot]_{0}^{L}$ indicates the boundary terms considered vanishing due to the specific boundary conditions. Then, imposing the stationarity for each independent variable variation, $\delta u, \delta v, \delta \theta, \delta \sigma_{\mathrm{x} 0}, \delta \sigma_{\mathrm{x} 1}$, and $\delta \tau$, the following six ODEs result:

$$
\begin{aligned}
& t^{\prime} \sigma_{x 0}+t \sigma_{x 0}^{\prime}+t f_{1}=0 \\
& 6\left(t^{\prime} c^{\prime}+t c^{\prime \prime}\right) \sigma_{x 0}+6 t c^{\prime} \sigma_{x 0}^{\prime}+3\left(t^{\prime 2}+t t^{\prime \prime}\right) \sigma_{x 1}+3 t t^{\prime} \sigma_{x 1}^{\prime}-4 t^{\prime} \tau-4 t \tau^{\prime}+6 t f_{2}=0 \\
& -t t^{\prime} \sigma_{x 1}+t^{2} \sigma_{x 1}^{\prime}+4 t \tau=0
\end{aligned}
$$




$$
\begin{aligned}
& -48 t u^{\prime}-48 t c^{\prime} v^{\prime}+\frac{t}{E}\left[48+48 c^{\prime 4}+(8-16 v) t^{\prime 2}+3 t^{\prime 4}+96 c^{2}+40 t^{\prime 2} c^{\prime 2}\right] \sigma_{x 0}+ \\
& \frac{16 t t^{\prime} c^{\prime}}{E}\left(4+4 c^{\prime 2}+t^{\prime 2}\right) \sigma_{x 1}-\frac{64 t}{E}(1+v) c^{\prime} \tau=0 \\
& 24 t t^{\prime} v^{\prime}+24 t t^{\prime} \theta+8 t^{2} \theta^{\prime}-\frac{16 t t^{\prime} c^{\prime}}{E}\left(4+4{c^{\prime}}^{2}+t^{\prime 2}\right) \sigma_{x 0}+\frac{32 t}{E}(1+v) t^{\prime} \tau- \\
& \frac{t}{E}\left[16+16 c^{\prime 4}+8(3+2 v) t^{\prime 2}+t^{\prime 4}+32 c^{\prime 2}+56 t^{\prime 2} c^{\prime 2}\right] \sigma_{x 1}=0 \\
& 5 t v^{\prime}+5 t \theta-\frac{10 t}{E}(1+v) c^{\prime} \sigma_{x 0}-\frac{5 t}{E}(1+v) t^{\prime} \sigma_{x 1}+\frac{8 t}{E}(1+v) \tau=0
\end{aligned}
$$

ODEs (24) are equipped with both natural and essential conditions, as clearly specified in Section 4.2. Moreover, we notice that the first three equations represent the equilibrium conditions: Equation (24a) expresses the equilibrium in the horizontal direction, Equation (24b) expresses the equilibrium in the vertical direction, and Equation (24c) expresses the rotational equilibrium. On the other hand, Equations (24d),(24e), and (24f) enforce the compatibility and the constitutive law equations, even though their physical meaning is not immediately clear.

\section{Non-prismatic beam examples}

In this section, we study three cases of non-prismatic cantilever beam with a concentrated load in the free edge and one case of non-prismatic clamped-clamped beam subject to a distributed body load in order to test the capabilities of the model proposed in Section 3.

Subsection 4.1 introduces the considered geometries, Subsection 4.2 specifies the different boundary conditions to be considered, Subsection 4.3 provides informations about the instruments to be used to evaluate both analytical and numerical solutions, and Subsection 4.4 illustrates and discusses the results.

\subsection{Geometry definition}

The considered cases are

- CASE1 symmetrically linear non-prismatic cantilever beam (see Figure 2),

- CASE2 symmetrically curvilinear non-prismatic cantilever beam (see Figure 3),

- CASE3 non-symmetrically curvilinear non-prismatic cantilever beam (see Figure 4),

- CASE4 non-symmetrically curvilinear non-prismatic clamped-clamped beam (see Figure 5).

For each case, Table 1 specifies the expressions of $c(x)$ and $t(x)$, which define the beam geometry. Substituting the expressions of $c(x)$ and $t(x)$ and their derivatives within Equation (24), it is possible to write the ODEs for each case. Their expressions are here omitted due to the derivation simplicity.

The symmetrically linear non-prismatic cantilever beam, shown in Figure 2, represents the simplest case to be discussed. Furthermore, the analytical expression of the stress distribution, for the considered geometry and load condition, is reported in $[21,11]$ and could be compared with the solution of the proposed analytical model.

The symmetrically curvilinear non-prismatic cantilever beam, shown in Figure 3, represents a particular case, known in literature as beam with uniform strength [10]. The geometry of this beam is such that its inertia varies along the beam-axis in a proportionally way with respect to the bending moment. This aspect leads to have the same maximum axial stress in each cross-section, which makes it convenient in terms of the amount of material used. Unlike the geometry proposed by Timoshenko [10], in Figure 3 the depth at the free edge of the beam is not equal to zero but sufficiently small to be considered negligible $(0.1 \mathrm{~m})$. In fact, the proposed analytical model does not work in the case of vanishing cross-sections for two reasons. The first one is that the cross-section area must be sufficiently big to transmit the shear force; the second one is that an horizontal outward unit vector implies a not defined stresses (see Equation (14)). 
Table 1: Analytical expressions of the centreline $c(x)$ and depth $t(x)$ for each considered example.

\begin{tabular}{lccc} 
EXAMPLES & $c(x)$ & $t(x)$ & $L / t(0)$ \\
\hline CASE1 & 0 & $-\frac{2 H}{L} x+4 H$ & 10 \\
CASE2 & 0 & $4 \sqrt{H^{2}\left(1-\frac{100 x}{101 L}\right)}$ & 10 \\
CASE3 & $-\frac{H(L-x)^{2}}{L^{2}}$ & $\frac{2 H\left(2 L^{2}-2 L x+x^{2}\right)}{L^{2}}$ & 5 \\
CASE4 & $-\frac{H(L-2 x)^{2}}{L^{2}}$ & $\frac{4 H\left(L^{2}-2 L x+2 x^{2}\right)}{L^{2}}$ & 10 \\
\hline
\end{tabular}

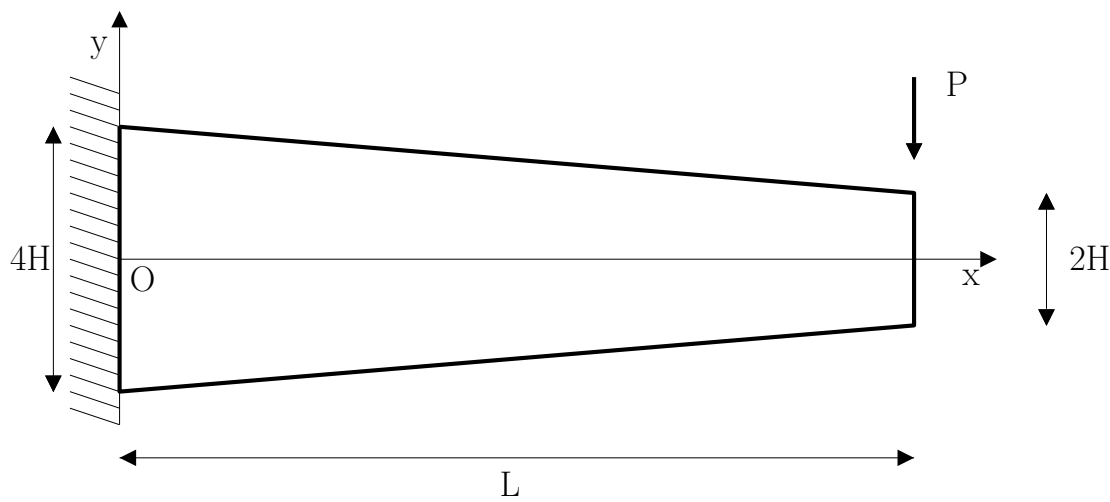

Figure 2: CASE1. Symmetrically linear non-prismatic cantilever beam, $H=0.25 \mathrm{~m}, L=10 \mathrm{~m}$, and $P=-100 \mathrm{kN}$

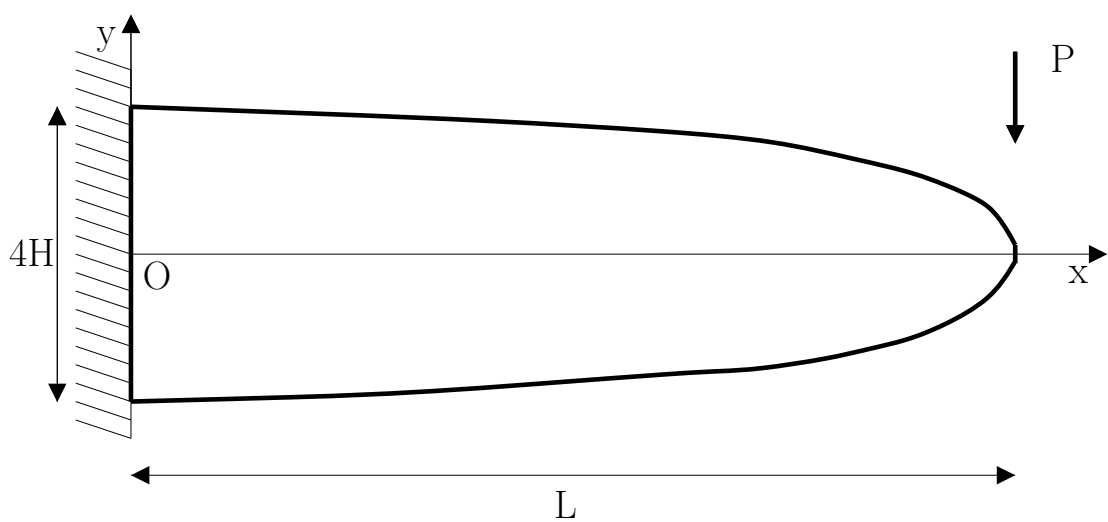

Figure 3: CASE2. Symmetrically curvilinear non-prismatic cantilever beam, $H=0.25 \mathrm{~m}, L=10 \mathrm{~m}$, and $P=-100 \mathrm{kN}$ 


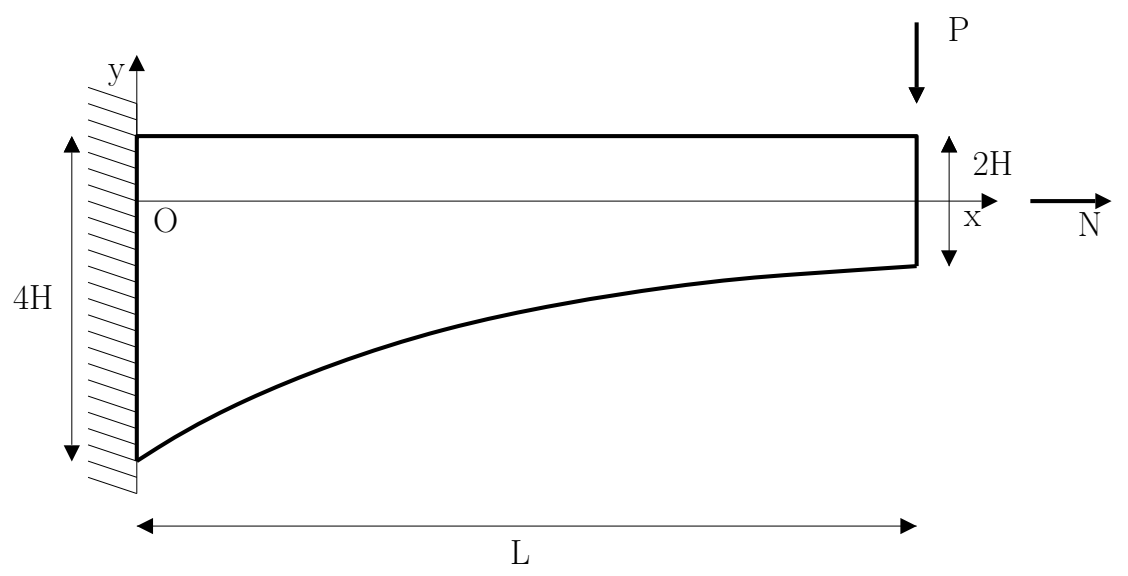

Figure 4: CASE3. Non-symmetrically curvilinear non-prismatic cantilever beam, $H=0.25 \mathrm{~m}, L=5 \mathrm{~m}, P=-100 \mathrm{kN}$, and $N=100 \mathrm{kN}$

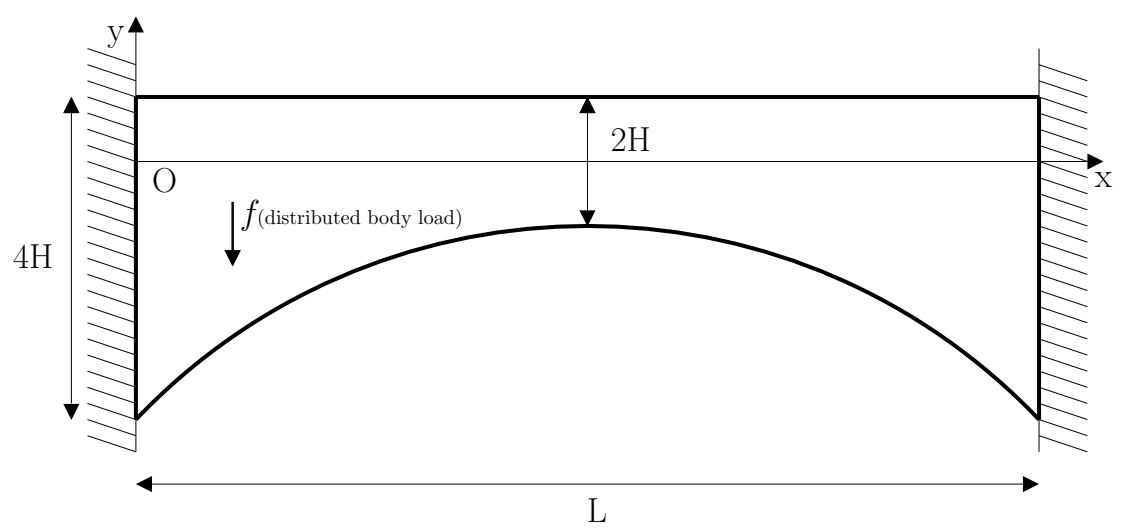

Figure 5: CASE4. Non-symmetrically curvilinear non-prismatic clamped-clamped beam, $H=0.25 \mathrm{~m}, L=10 \mathrm{~m}$, and $f_{2}=-50 \mathrm{kN} / \mathrm{m}^{2}$ 
The non-symmetrically curvilinear non-prismatic cantilever beam , shown in Figure 4, presents a more complex geometry. In fact, the beam is not symmetric (i.e. $c(x)$ is not a constant function), and less slender (see the parameter $L / t(0)$ in Table 1). Moreover, for this case we separately consider two different load conditions: a transversal concentrated load and an axial concentrated load in order to prove that the model naturally takes the flexural and extensional coupling into account without further model modifications.

In the end, the non-symmetrically curvilinear non-prismatic clamped-clamped beam (see Figure 5) shows the capability of the proposed model to yield results even in cases of statically indeterminate (or hyperstatic) beams and distributed loads.

\subsection{Boundary conditions and material properties}

Focusing on Figures 2, 3, and 4, the beams are clamped at $x=0$ and subject to a transversal concentrated load $P$ equal to $-100 \mathrm{kN}$, at $x=L$. Moreover, the distributed body load vector $\boldsymbol{f}$ has both components $f_{1}$ and $f_{2}$ equal to 0 .

In order to solve ODEs (24) for CASE1, CASE2, and CASE3, the following boundary conditions are imposed:

$$
\begin{array}{ll}
\theta(0)=0 & \sigma_{x 0}(L)=0 \\
v(0)=0 & \sigma_{x 1}(L)=0 \\
u(0)=0 & \tau(L)=-(3 P) /(2 h)
\end{array}
$$

where $h$ represents the beam depth at the free edge.

For the non-symmetrically curvilinear non-prismatic cantilever beam, CASE3 (see Figure 4), an axial concentrated load case with $N$ equal to $100 \mathrm{kN}$ is also considered, using the following boundary conditions:

$$
\begin{array}{ll}
\theta(0)=0 & \sigma_{x 0}(L)=N / h \\
v(0)=0 & \sigma_{x 1}(L)=0 \\
u(0)=0 & \tau(L)=0
\end{array}
$$

A different choice is made for the hyperstatic beam by applying a distributed body load vector on the whole domain $\Omega$ (see Figure 5). Therefore, $f_{1}$ is equal to 0 and $f_{2}$ is assumed equal to $-50 \mathrm{kN} / \mathrm{m}^{2}$. The boundary conditions related to CASE4 are listed hereunder:

$$
\begin{array}{ll}
\theta(0)=0 & \theta(L)=0 \\
v(0)=0 & v(L)=0 \\
u(0)=0 & u(L)=0
\end{array}
$$

Finally, the following values of Young's modulus $E$ and Poisson's ratio $v$ as material parameters are assumed:

$$
\begin{aligned}
& E=10 \cdot 10^{7} \mathrm{kN} / \mathrm{m}^{2} \\
& v=0.3
\end{aligned}
$$

\subsection{Considered models and control parameters}

In order to test the proposed analytical model, we consider the following quantities as control parameters:

1. the transversal-displacement evaluated at the free edge, $v(L)$, and its distribution along the beam longitudinal axis;

2. the maximum value of the shear stress at half length, $\max \left(\sigma_{x y}\right)$, and the shear stress distribution within the same cross-section; 
3. the maximum value of the horizontal displacement at the free edge, $\max _{y \in A(L)}\left(u_{s}\right)$, and the horizontal displacement distribution within the same cross-section;

4. the maximum value of the horizontal stress at half length, $\max _{y \in A(L / 2)}\left(\sigma_{x x}\right)$, and the horizontal stress distribution within the same cross-section;

5. the transversal-displacement evaluated at half length, $v(L / 2)$, and its distribution along the beam longitudinal axis;

6. the maximum value of the shear stress at one quarter of length, $\max _{y \in A(L / 4)}\left(\sigma_{x y}\right)$, and the shear stress distribution within the same cross-section.

In particular, the parameters defined in 1 and 2 are considered for CASE1, CASE2, and CASE3, the parameters in 3 and 4 are additional control parameters for axial load condition presented in CASE3, whereas 5 and 6 are used for CASE4 only.

In the following, the models, through which is possible to evaluate the aforementioned parameters and distributions, are listed.

- Euler-Bernoulli beam theory (EB). The Principle of Virtual Work (PVW) is used together with the hypotheses of Euler-Bernoulli beam theory to find $v(L)$ and to construct the transversal-displacement distribution along the beam longitudinal axis. According to the boundary conditions illustrated in Equation (25), the PVW can be written as follows:

$$
v(x)=\int_{0}^{L}\left[(L-x) \frac{P(L-x)}{E I(x)}\right] d x
$$

where $I(x)$ represents the second moment of area, which is assumed to vary along the beam-axis.

- Timoshenko beam theory (T). The PVW is used together with the hypotheses of Timoshenko beam theory to find $v(L)$ and to construct the transversal-displacement distribution along the beam longitudinal axis. According to the boundary conditions illustrated in Equation (25) the PVW can be written as follows:

$$
v(x)=\int_{0}^{L}\left[(L-x) \frac{P(L-x)}{E I(x)}\right] d x+\int_{0}^{L}\left[\frac{P}{(5 / 6) G t(x)}\right] d x
$$

where $G$ represents the shear modulus, defined as $G=\frac{E}{2(1+v)}$.

- Jourawsky theory $(\mathbf{J})$. The Jourawsky theory is used to find $\max _{y \in A(L / 2)}\left(\sigma_{x y}\right)$ and to construct the shear stress distribution at half length of the beam.

- Analytical model (AN). Once the analytical expression of the ODEs is obtained, the solution for each beam can be calculated by means of the Mathematica command NDSolve [Mathematica version 7 30] and by imposing the boundary conditions specified in Section 4.2. We decide to use the command NDSolve instead of an analytical procedure, since it is possible to obtain the solution with a more reasonable computational time (CPU time: 60 seconds, Intel(R) Core(TM) i5 CPU, 4 GB of RAM, operating system 64 bit).

- FE analysis (FE). 2D FE analyses are performed through the software ABAQUS [31], using the abovedescribed beam geometries and mechanical parameters, $E$ and $v$. Then, for CASE1, CASE2, and CASE3 a fixed support at the left edge of the beam and a boundary load at the right edge, modelled as a "Surface Traction" load, are enforced, whereas for CASE4 a fixed support in both edges of the beam and a distributed body load directing downwards, modelled as "Body Force", are enforced. Lastly, an appropriate quadrangular mesh characterized by an approximate element size of $0.05 \mathrm{~m}$ is generated, arranged by $\sim 3000$ elements. This choice is the result of an accurate convergence analysis, which ensured that the numerical error introduced by the considered geometry discretization is smaller than the number of digits used to report the results, leading further computational efforts to be useless. 
In the following sections, the FE solution is considered the reference solution. Therefore, the errors can be estimated by using the expression hereunder:

$$
e_{q}=\frac{\left|q-q_{F E}\right|}{\left|q_{F E}\right|}
$$

where $q$ indicates the considered quantity.

\subsection{Numerical results}

This section illustrates the obtained results and discusses them in order to highlight the main advantages and limitations of the proposed model.

\subsubsection{Symmetrically linear non-prismatic cantilever}

Table 2 illustrates the results for the symmetrically linear non-prismatic cantilever beam, depicted in Figure 2 . In particular, the transversal-displacement at the free edge of the beam, $v(L)$, the maximum value of the shear stress at half length of the beam, $\max _{y \in A(L / 2)}\left(\sigma_{x y}\right)$, and their percentage errors, $e_{v}$ and $e_{\sigma_{x y}}$, are reported. It is worth noticing that the transversal-displacement results are quite similar to the reference solution (FE) with a percentage error less than $0.5 \%$ for all methods. Furthermore, Figure 6 shows a high degree of matching among the transversal-displacement distributions.

Table 2: Results for the symmetrical linear non-prismatic cantilever beam.

\begin{tabular}{lcccc} 
METHODS & $v(L)[\mathrm{m}]$ & $\max _{y \in A(L / 2)}\left(\sigma_{x y}\right)\left[\mathrm{kN} / \mathrm{m}^{2}\right]$ & $e_{v}[\%]$ & $e_{\sigma_{x y}}[\%]$ \\
\hline EB & $-6.542 \cdot 10^{-3}$ & - & $4.295 \cdot 10^{-1}$ & - \\
T & $-6.585 \cdot 10^{-3}$ & - & $2.288 \cdot 10^{-1}$ & - \\
J & - & $-2.000 \cdot 10^{2}$ & - & $4.993 \cdot 10^{1}$ \\
AN & $-6.577 \cdot 10^{-3}$ & $-1.333 \cdot 10^{2}$ & $1.012 \cdot 10^{-1}$ & $4.648 \cdot 10^{-2}$ \\
FE & $-6.570 \cdot 10^{-3}$ & $-1.334 \cdot 10^{2}$ & 0 & 0 \\
\hline
\end{tabular}

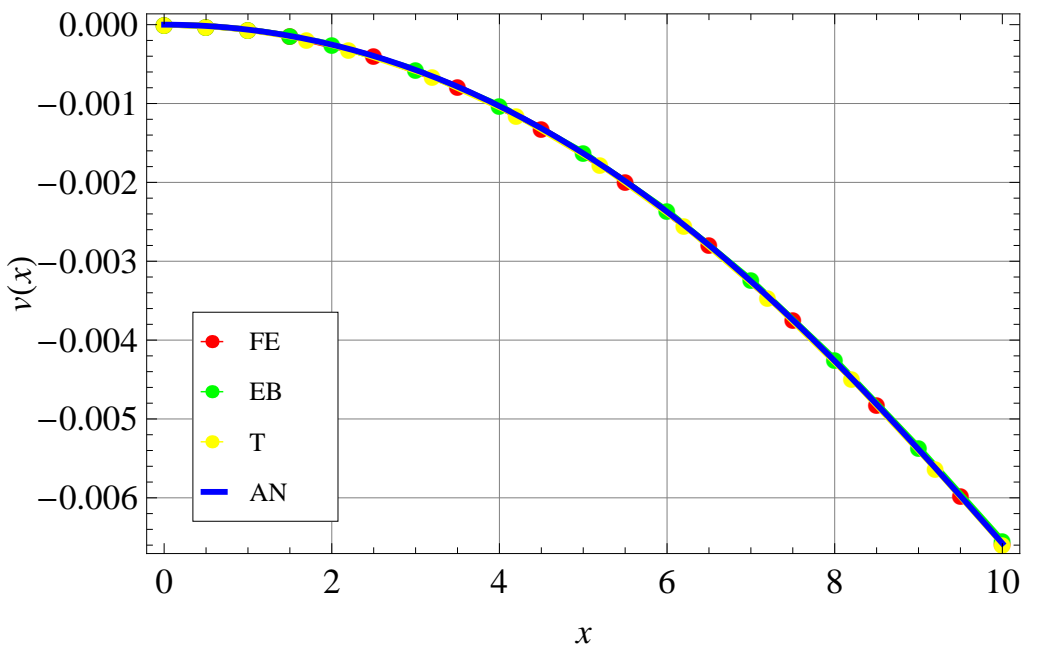

Figure 6: CASE1. Transversal-displacement distributions.

With regard to the shear stress, there is a clear mismatch between the Jourawsky solution (J) and the other two solutions (FE and AN). As shown in Table 2, the percentage error of $\mathrm{J}$ is up to 50\%, whereas the percentage error 


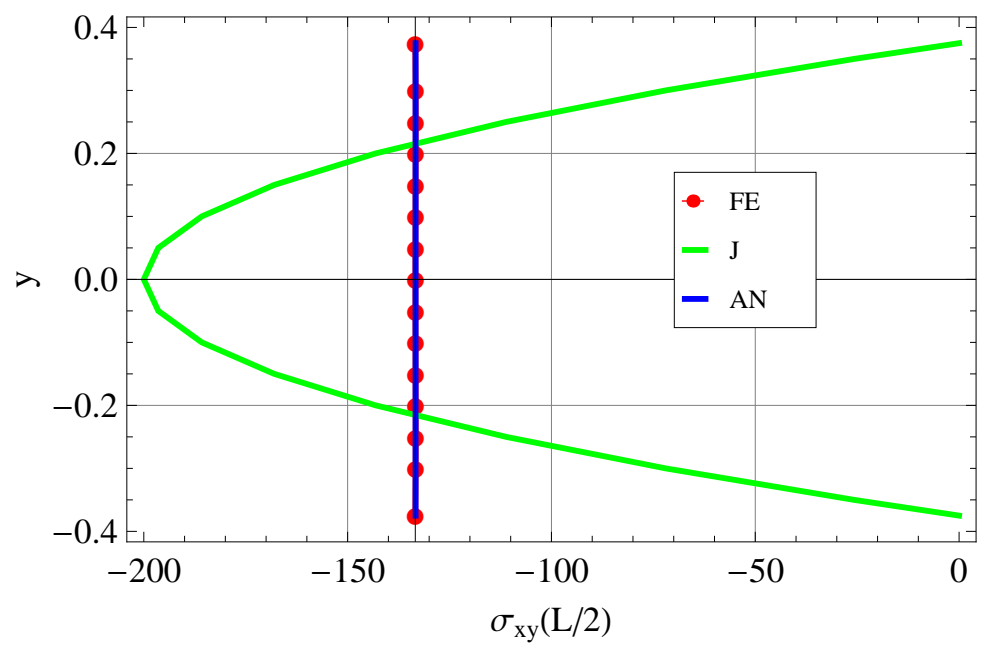

Figure 7: CASE1. Shear stress distributions at half length of the beam.

of $\mathrm{AN}$ is less than $0.05 \%$, indicating the high accuracy of the developed model in predicting stresses. Figure 7 emphasizes the mismatch between $\mathbf{J}$ and AN. In fact, the shear stress distribution of $\mathbf{J}$ is parabolic, on the contrary the shear distributions of FE and AN are constant over the whole cross-section. It is worth noticing that this counterintuitive result -already discussed in [32]- is a consequence of the non-trivial dependence of stress distributions on beam geometry and applied load.

\subsubsection{Symmetrically curvilinear non-prismatic cantilever}

Table 3 compares results for symmetrically curvilinear non-prismatic cantilever beam, depicted in Figure 3 . All methods provide a value of the transversal-displacement, at the free edge of the beam, very similar to the reference solution (FE) with a percentage errors less than $1 \%$. Furthermore, Figure 8 shows a high degree of matching among all transversal-displacement distributions.

Table 3: Results for the symmetrically curvilinear non-prismatic cantilever beam.

\begin{tabular}{lcccc} 
METHODS & $v(L)[\mathrm{m}]$ & $\max _{y \in A(L / 2)}\left(\sigma_{x y}\right)\left[\mathrm{kN} / \mathrm{m}^{2}\right]$ & $e_{v}[\%]$ & $e_{\sigma_{x y}}[\%]$ \\
\hline & & & & \\
EB & $-7.815 \cdot 10^{-3}$ & - & $9.631 \cdot 10^{-1}$ & - \\
T & $-7.872 \cdot 10^{-3}$ & - & $2.407 \cdot 10^{-1}$ & - \\
J & - & $-2.111 \cdot 10^{2}$ & - & $9.440 \cdot 10^{1}$ \\
AN & $-7.857 \cdot 10^{-3}$ & $-1.076 \cdot 10^{2}$ & $4.309 \cdot 10^{-1}$ & $8.605 \cdot 10^{-1}$ \\
FE & $-7.891 \cdot 10^{-3}$ & $-1.085 \cdot 10^{2}$ & 0 & 0 \\
\hline
\end{tabular}

Considering the shear stress results, there is again a clear mismatch between the Jourawsky solution $(\mathrm{J})$ and the other two solutions (FE and AN). In Figure 9, the J shear stress distribution has an opposite concavity with respect to the concavity of FE and AN shear stress distributions. The percentage error of $\mathrm{J}$ is around $100 \%$, whereas the percentage error of AN is around 1\% (see Table 3). Therefore, we prove that the proposed model (AN) has a much higher accuracy than Jourawsky formula, which fails in the case of non-prismatic beams. Moreover, Figure 9 highlights that the shear stress is generally different from zero on the upper and lower limits of the domain, contrary to the usual shear stress distributions in prismatic beams. The main reason is that, when the beam presents a variable cross-section, the shear stress on the upper and lower limits of the domain must be different from zero in order to satisfy the boundary equilibrium (see Equation (14)). 


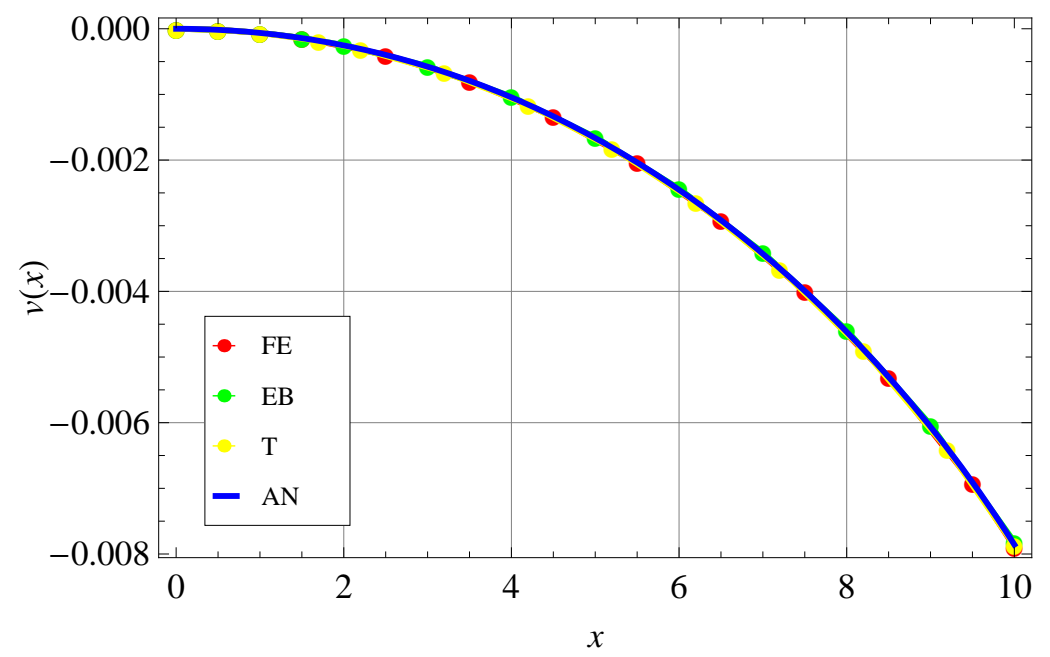

Figure 8: CASE2. Transversal-displacement distributions.

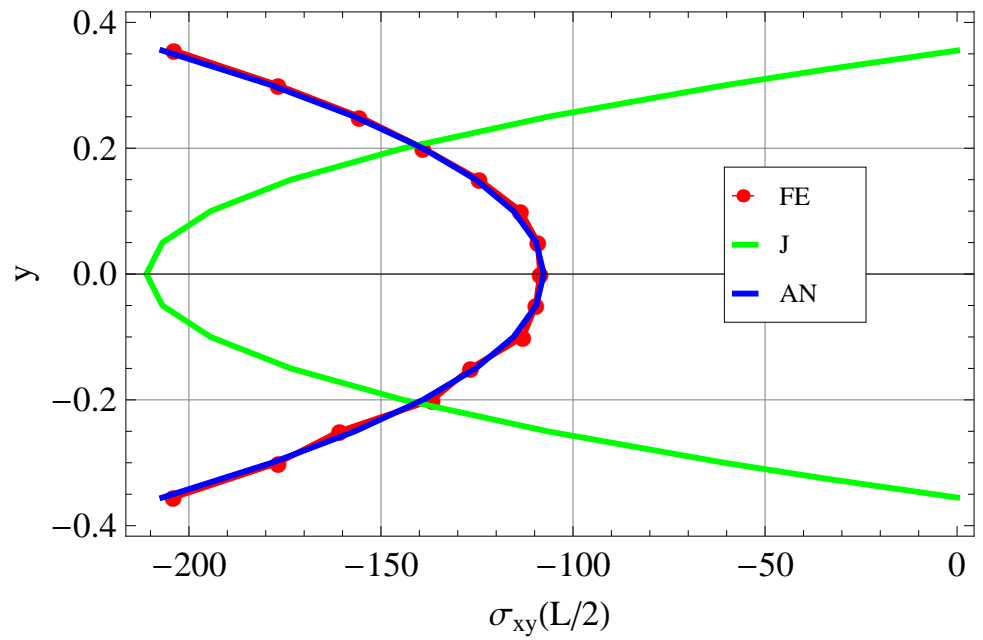

Figure 9: CASE2. Shear stress distributions at half length of the beam 


\subsubsection{Non-symmetrically curvilinear non-prismatic cantilever}

For the non-symmetrically curvilinear non-prismatic cantilever beam, shown in Figure 4, two load conditions, separately applied at the free end, are considered: a transversal concentrated load and an axial one. The comparison results for transversal concentrated load is reported in Table 4. It is worth noticing that the proposed model (AN) shows a good matching with the reference solution $(\mathrm{FE})$ in both transversal-displacement and shear stress distributions. The errors, $e_{v}$ and $e_{\sigma_{x v}}$, are around $0.2 \%$ and $0.5 \%$, respectively. Moreover, the high degree of matching is also proven in Figure 10.

Table 4: Results for the non-symmetrically curvilinear non-prismatic cantilever beam subject to the transversal concentrated load $P$

\begin{tabular}{|c|c|c|c|c|}
\hline METHODS & $v(L)[\mathrm{m}]$ & $\max _{y \in A(L / 2)}\left(\sigma_{x y}\right)\left[\mathrm{kN} / \mathrm{m}^{2}\right]$ & $e_{v}[\%]$ & $e_{\sigma_{x y}}[\%]$ \\
\hline EB & $-1.178 \cdot 10^{-3}$ & - & $2.394 \cdot 10^{0}$ & - \\
\hline $\mathrm{T}$ & $-1.203 \cdot 10^{-3}$ & - & $3.645 \cdot 10^{-1}$ & - \\
\hline $\mathrm{J}$ & - & $-2.400 \cdot 10^{2}$ & - & $3.784 \cdot 10^{1}$ \\
\hline AN & $-1.210 \cdot 10^{-3}$ & $-3.840 \cdot 10^{2}$ & $2.519 \cdot 10^{-1}$ & $5.496 \cdot 10^{-1}$ \\
\hline FE & $-1.207 \cdot 10^{-3}$ & $-3.861 \cdot 10^{2}$ & 0 & 0 \\
\hline
\end{tabular}

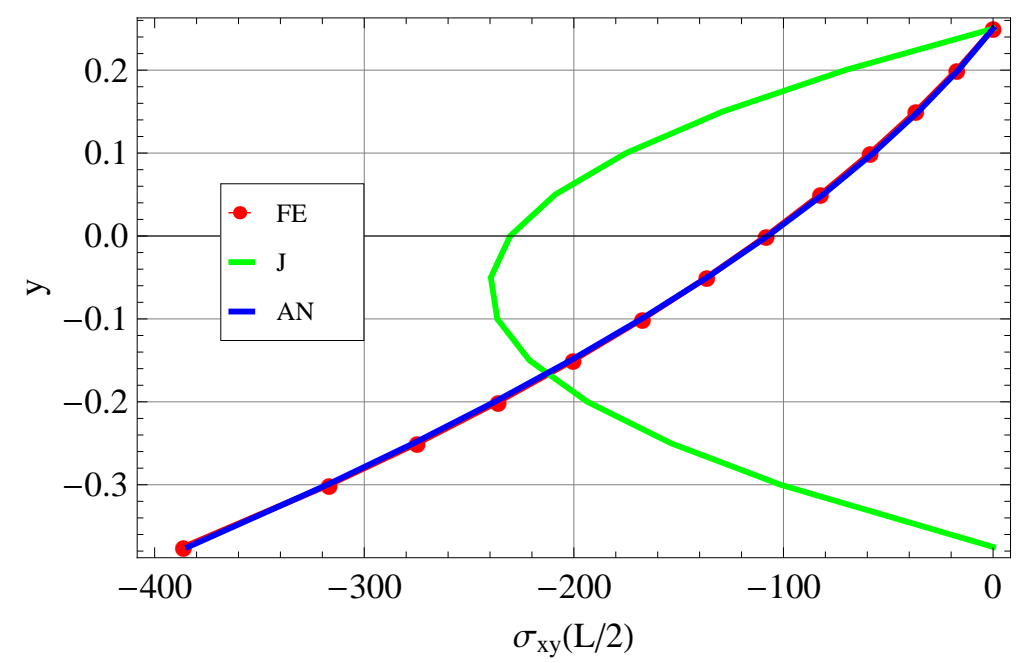

Figure 10: CASE3. Shear stress distributions at half length of the beam determined by load $P$.

Table 5 refers to the case of axial concentrated load. In particular, the transversal-displacement at the free edge of the beam, $v(L)$, the maximum value of the shear stress at half length of the beam, $\max _{y \in A(L / 2)}\left(\sigma_{x y}\right)$, the maximum value of the horizontal displacement at the free edge of the beam, $\max _{y \in A(L)}\left(u_{s}\right)$, the maximum value of the horizontal stress at half length of the beam, $\max _{y \in A(L / 2)}\left(\sigma_{x x}\right)$, and their percentage errors, $e_{v} e_{\sigma_{x y}} e_{u}$ and $e_{\sigma_{x x}}$, are reported. We do not introduce $\mathrm{EB}$ and $\mathrm{T}$ rows because it is standard to neglect coupling, assuming a vanishing value of transversal displacement, i.e. $v(L)=0$. Once more, AN shows a good matching with the reference solution with a percentage error around $2 \%$, which is acceptable in the most of practical engineering applications.

Figures 11, 12 and 13, highlight a high degree of matching between the AN displacement and stress distributions and the FE ones, indicating that the proposed model is also suitable for the study of non-symmetric non-prismatic beam. Furthermore, we highlight that the proposed method has the capability to take the flexural and extensional coupling into account without needing any further model manipulations or calibrations. 
Table 5: Results for the non-symmetrically curvilinear non-prismatic cantilever beam subject to the axial concentrated load $N$

\begin{tabular}{lcccc} 
METHODS & $v(L)[\mathrm{m}]$ & $\max _{y \in A(L / 2)}\left(\sigma_{x y}\right)\left[\mathrm{kN} / \mathrm{m}^{2}\right]$ & $e_{v}[\%]$ & $e_{\sigma_{x y}}[\%]$ \\
\hline AN & $-3.780 \cdot 10^{-5}$ & $6.400 \cdot 10^{0}$ & $1.547 \cdot 10^{-1}$ & $2.259 \cdot 10^{0}$ \\
FE & $-3.774 \cdot 10^{-5}$ & $6.259 \cdot 10^{0}$ & 0 & 0 \\
\hline \multirow{2}{*}{ METHODS } & $\max _{y \in A(L)}\left(u_{s}\right)[\mathrm{m}]$ & $\max _{y \in A(L / 2)}\left(\sigma_{x x}\right)\left[\mathrm{kN} / \mathrm{m}^{2}\right]$ & $e_{u}[\%]$ & $e_{\sigma_{x x}}[\%]$ \\
\hline AN & $1.215 \cdot 10^{-5}$ & $2.560 \cdot 10^{2}$ & $2.508 \cdot 10^{-1}$ & $1.875 \cdot 10^{-1}$ \\
FE & $1.212 \cdot 10^{-5}$ & $2.555 \cdot 10^{2}$ & 0 & 0 \\
\hline
\end{tabular}

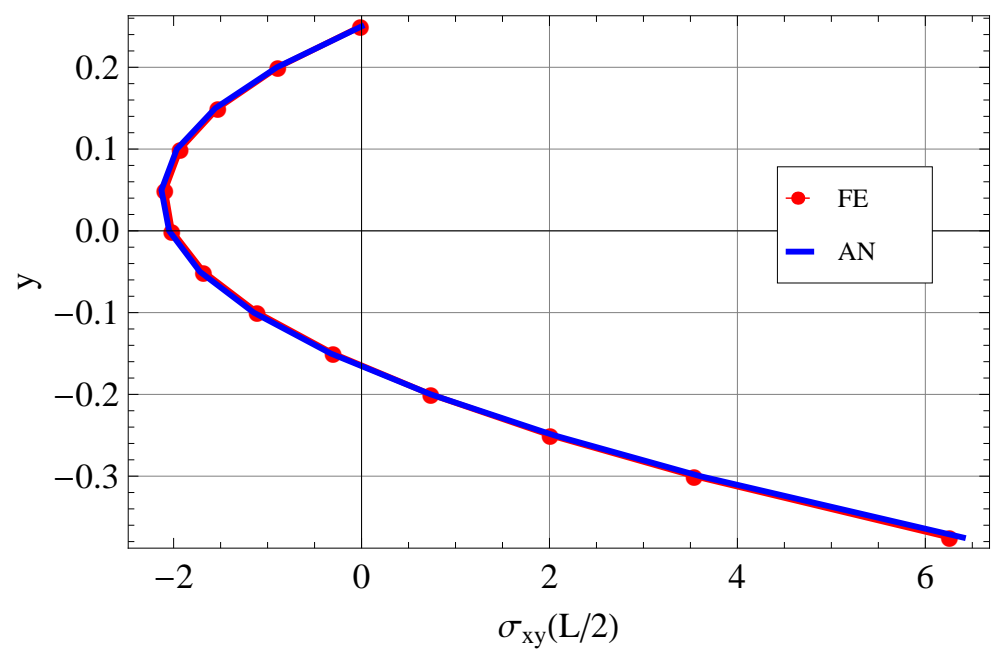

Figure 11: CASE3. Shear stress distributions at half length of the beam determined by load $N$.

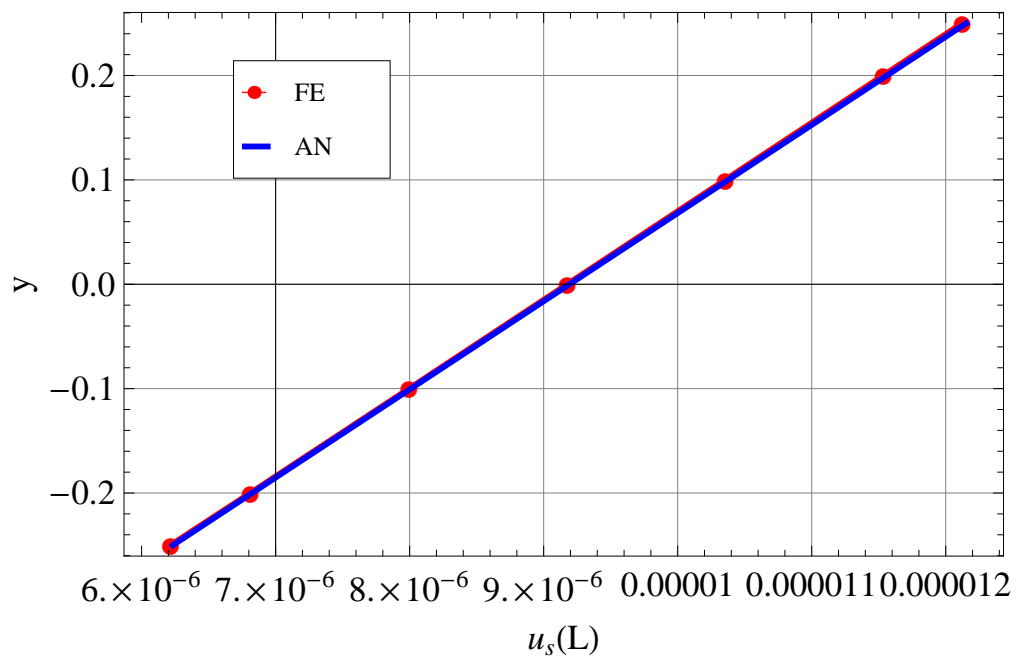

Figure 12: CASE3. Horizontal displacement distributions at the free edge of the beam determined by load $N$. 


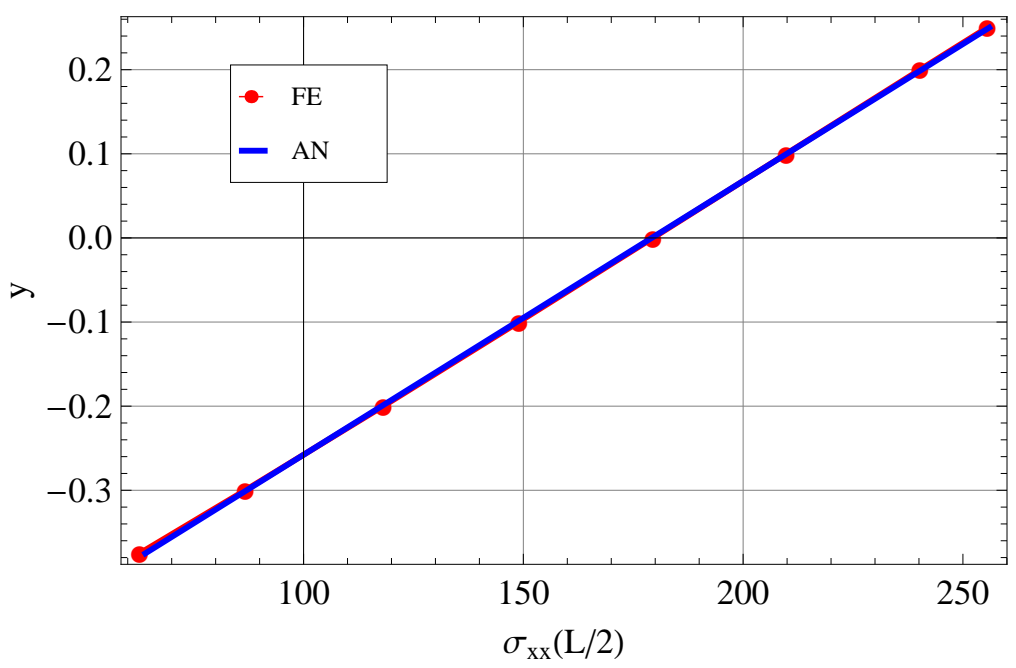

Figure 13: CASE3. Horizontal stress distributions at half length of the beam determined by load $N$.

\subsubsection{Non-symmetrically curvilinear non-prismatic clamped-clamped}

Table 6 illustrates the results for the non-symmetrically curvilinear non-prismatic clamped-clamped beam, depicted in Figure 5. For this case, the transversal-displacement evaluated at half length of the beam, the maximum value of the shear stress at one quarter of length, $\max _{y \in A(L / 4)}\left(\sigma_{x y}\right)$, and their percentage errors, $e_{v}$ and $e_{\sigma_{x y}}$, are reported. Moreover, we evaluate the horizontal reactions at the two edges, $N(0)=\int_{A(0)} \sigma_{x x} d y$ and $N(L)=\int_{A(L)} \sigma_{x x} d y$. The results remark once more that the proposed model catches correctly the coupling occurring between axial and flexural equations.

It is worth noticing that the transversal-displacement result, obtained by using AN, is quite similar to the reference solution (FE) with a percentage error less than 3\%, acceptable in the most of practical engineering applications. The high degree of matching between the transversal-displacement distributions is also shown in Figure 14.

With regard to the shear stress, the percentage error is around 1.5\% (see Table 6 and Figure 15), proving that the model catches accurate results even in cases of hyperstatic non-prismatic beam.

Table 6: Results for the non-symmetrically curvilinear non-prismatic clamped-clamped beam subject to the transversal concentrated load $P$

\begin{tabular}{lcccccc} 
METHODS & $v(L / 2)[\mathrm{m}]$ & $\max _{y \in A(L / 4)}\left(\sigma_{x y}\right)\left[\mathrm{kN} / \mathrm{m}^{2}\right]$ & $e_{v}[\%]$ & $e_{\sigma_{x y}}[\%]$ & $N(0)[\mathrm{kN}]$ & $N(L)[\mathrm{kN}]$ \\
\hline AN & $-2.314 \cdot 10^{-4}$ & $-1.514 \cdot 10^{2}$ & $2.753 \cdot 10^{0}$ & $1.317 \cdot 10^{0}$ & -131.543 & -131.543 \\
FE & $-2.252 \cdot 10^{-4}$ & $-1.494 \cdot 10^{2}$ & 0 & 0 & -131.233 & -131.233 \\
\hline
\end{tabular}

\subsubsection{Final remarks}

Figures 16 and 17 summarize the results introduced in Section 4.4 for CASE1, CASE2, and CASE3 (beam subject to load $P$ ).

In particular, Figure 16 compares the percentage errors related to the transversal-displacement results at the free edge of the non-prismatic cantilever beams. The green columns refers to EB, the yellow columns to T, and the blue columns to AN. It is worth noticing that the proposed model presents much higher accuracy than EB and similar accuracy to T. In particular, for CASE3, the EB model shows the worst error, maybe because the beam is less slender with respect to the other cases.

It is worth noting that, Equation (30), resulting from the Timoshenko beam theory, considers a shear correction factor equal to 5/6. On the contrary, Figures 7 and 9 highlight that the stress distribution does no longer correspond to 


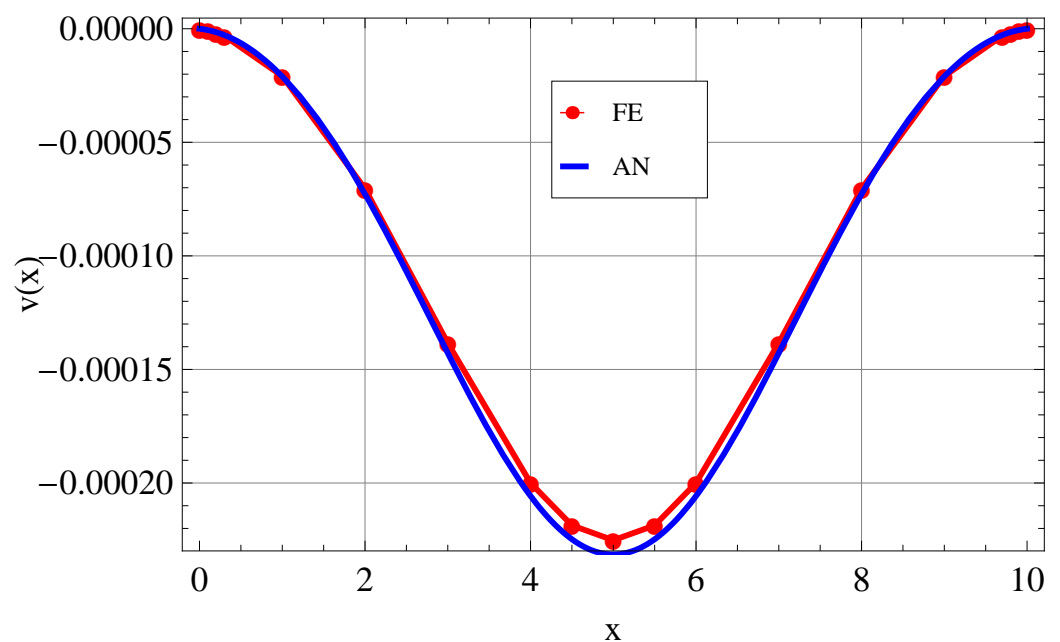

Figure 14: CASE4. Transversal-displacement distributions.

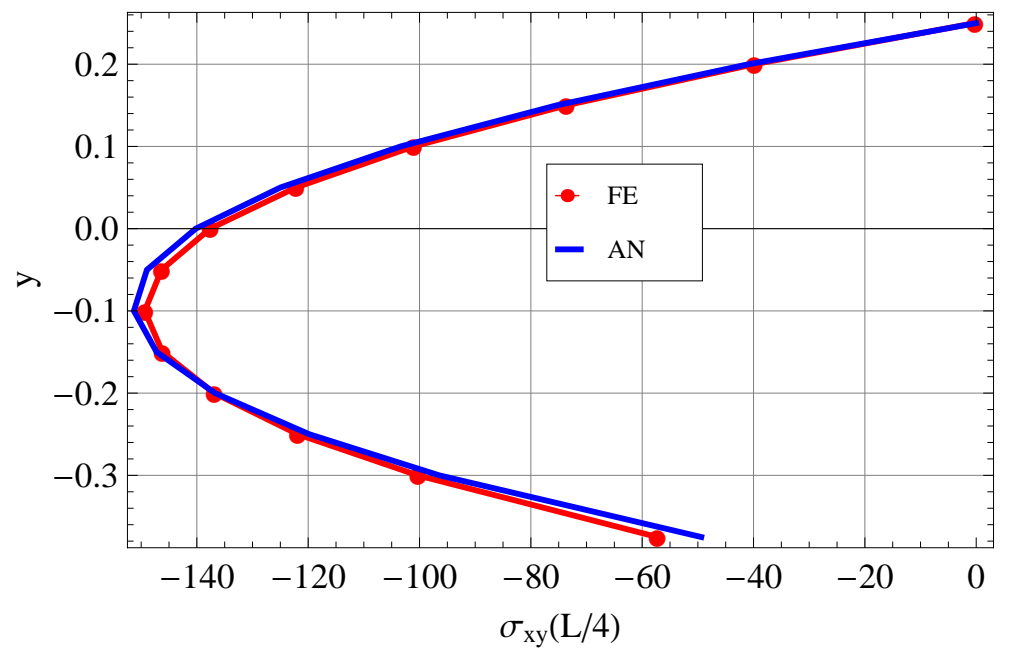

Figure 15: CASE4. Shear stress distributions at one quarter of beam length. 


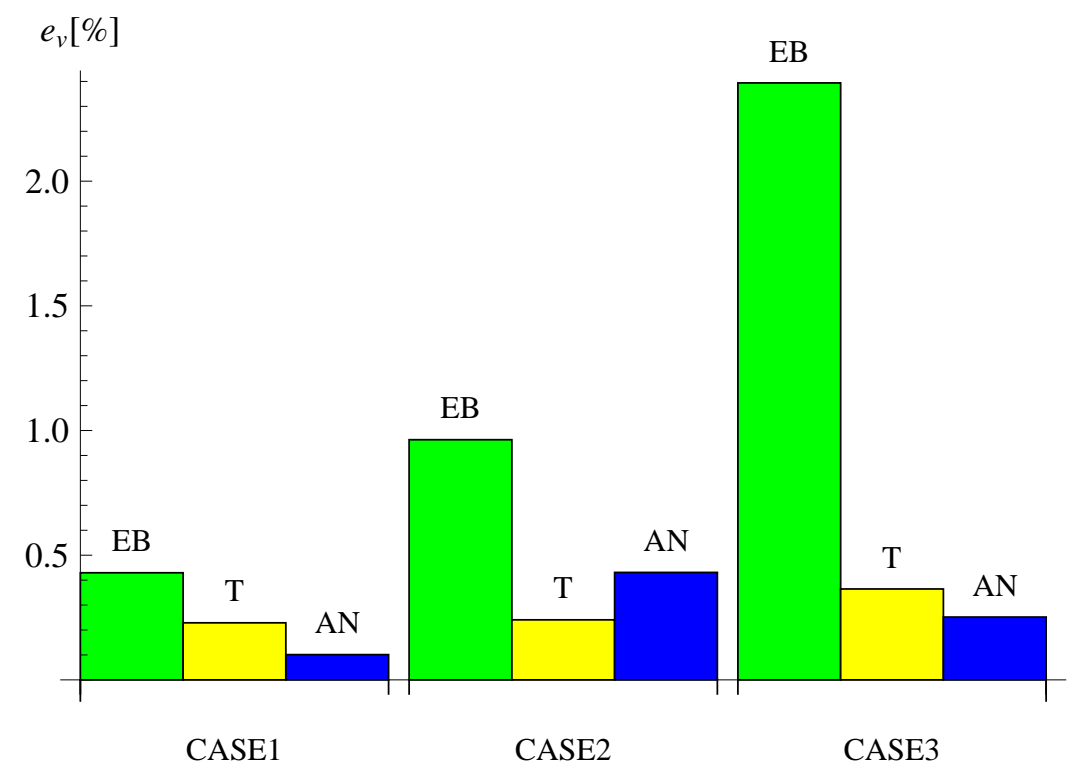

Figure 16: Errors related to the transversal-displacement results.

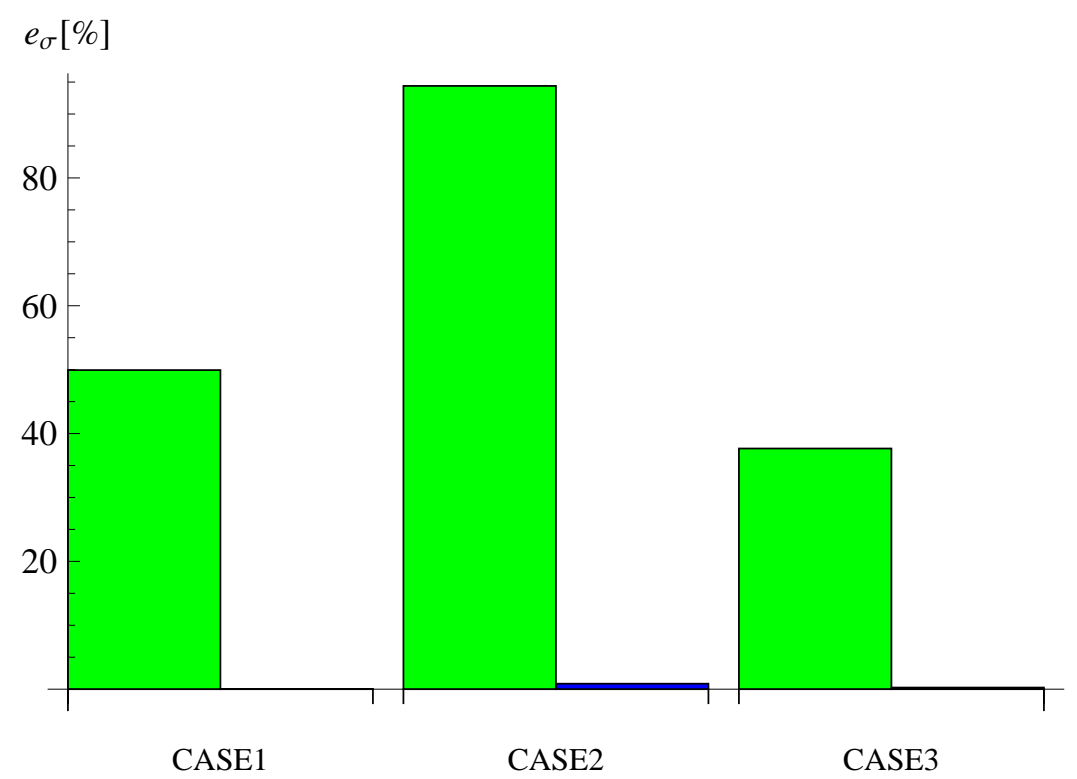

Figure 17: Errors related to the shear stress results. 
the usual Jourawsky profile, indirectly proving a variation of the shear correction factor along the beam axis. To this end, an important advantage of the proposed analytical model is its natural capability of capturing the shear correction factor variation. Therefore, a further development of the present work could be the investigation into the effects of the lateral surface slope on the shear correction factor and its explicit evaluation for a generic non-prismatic beam.

Moreover, both Equations 29 (in EB hypotheses) and 30 (in T hypotheses) are not able to distinguish the case of a symmetrically non-prismatic beam from the case of a non-symmetrically one. For instance, considering the boundary conditions 25 for the study of two linearly non-prismatic beams, respectively symmetric and non-symmetric, with the same length and same cross-section area at the two edges, Equations 29 and 30 provide the same transversaldisplacement even though it could be very different. The reason is that Equations 29 and 30 are not able to capture the fact that all cross-section centroids belong to the beam-axis (condition verified in the symmetric case only), but they only account for the cross-section variation due to the dependence on $x$ of the area and of the second moment of area. On the contrary, the proposed analytical model (AN) naturally overcomes the aforementioned limitation and predicts transversal-displacements with a high degree of accuracy.

With regard to stresses, Figure 17 compares relative errors of shear stress, specifically the green columns are related to $\mathrm{J}$, and the blue ones to AN. It is evident how the analytical model can calculate the shear stress distribution with high accuracy, which is another advantage over Euler-Bernoulli and Timoshenko beam theories. Moreover, it is clear that $\mathrm{AN}$ achieves much higher accuracy than $\mathrm{J}$ and its mismatch with the reference solution (FE) is less than $1 \%$ in all cases.

All the remarks can be also referred to the more complicated study of indeterminate beam discussed in Section 4.4.3.

In conclusion, the proposed model is able to reach a very good accuracy in terms of stress and displacement distributions because it provides results as accurate as the ones obtained by using more expensive 2D FE analyses. Furthermore, since the proposed model consists of six ODEs, whose solution could be evaluated through standard analytical and numerical tools, it represents a good compromise between computational efficiency and solution accuracy.

\section{Conclusions}

The study conducted in this paper allowed the modelling of beams with complex geometries through simple kinematic assumptions, guaranteeing highly accurate stress results nonetheless.

In Sections 2 and 3, we presented the general derivation of the proposed analytical beam model, obtaining six ODEs suitable for studying a generic 2D linear-elastic non-prismatic beam. In section 4, we focused on three examples of cantilever non-prismatic beam with a concentrated load at the free edge and one example of clamped-clamped beam subject to a distributed body load. The result comparison shows that the analytical model provides results with percentage errors around $1 \%$ in isostatic case and around $3 \%$ in hyperstatic case, even though the number of un-known variables is substantially lower than the FE analysis (reference solution).

This model can be easily generalized to the study of more complex problems, such as non-homogeneous nonprismatic beams, 3D beams and beams characterized by and/or non-linear constitutive laws, due to the absence of limiting hypotheses in the formulation. Lastly, the possibility to derive a suitable FE analysis, as suggested by [23], will allow its integration within existing structural analysis software.

\section{Acknowledgements}

This work was partially funded by Cariplo Foundation through the Project SICURA and by MIUR (Italian Department of University Research) through the Project PRIN 2010-2011.

[1] Portland Cement Association. Handbook of frame constants, 1958.

[2] Arturo Tena Colunga. Stiffness formulation for nonprismatic beam elements. Journal of Structural Engineering, 122(12):1484-1489, 1996.

[3] Can Balkaya and Ergin Citipitioglu. Discusson of the paper "Stiffness formulation for nonprismatic beam elements" by Arture Tena-Colunga. Journal of structural engineering, 123(12):1694-1695, 1997.

[4] Can Balkaya. Behavior and modeling of nonprismatic members having T-sections. Journal of structural engineering, 127:940-946, 2001.

[5] J. R. Banerjee and F. W. Williams. Exact bernoulli-euler static stiffness matrix for a range of tapered beam-columns. International Journal for Numerical Methods in Engineering, 23(9):1615-1628, 1986. 
[6] Z. Friedman and J.B. Kosmatka. Exact stiffness matrix of a nonuniform beam-i. extension, torsion, and bending of a bernoulli-euler beam. Computers $\mathcal{E}$ Structures, 42(5):671 - 682, 1992.

[7] Giray Ozay and Ahmet Topcu. Analysis of frames with non-prismatic members. Canadian Journal of Civil Engineering, 27:17-25, 2000.

[8] B. A. Boley. On the Accuracy of the Bernoulli-Euler Theory for Beams of Variable Section. Journal of Applied Mechanics, 30:373, 1963.

[9] S.P. Timoshenko. Strength of Materials: Part II Advanced Theory and Prblems. D. Van Nostrand, 1963.

[10] S. Timoshenko. Strength of Materials: Elementary theory and problems. Strength of Materials. R. E. Krieger Pub. Co., 1976.

[11] JL Krahula. Shear formula for beams of variable cross section. AIAA Journal, 13:1390-1391, 1975.

[12] Dewey Hodges, Anurag Rajagopal, Jimmy Ho, and Wenbin Yu. Stress and strain recovery for the in-plane deformation of an isotropic tapered strip-beam. Journal of Mechanics of Materials and Structures, 5(6):963-975, 2011.

[13] S. Timoshenko and J.N. Goodier. Theory of Elasticity, by S. Timoshenko and J. N. Goodier,... 2nd Edition. McGraw-Hill book Company, 1951.

[14] Friedrich Bleich. Stahlhochbauten: Ihre Theorie, Berechnung Und Bauliche Gestaltung. Springer, 1932.

[15] American Concrete Institute. Building code requirements for structural concrete (ACI 318-05) and commentary (ACI 318R-05), 2005.

[16] European Committee for Standardisation. EN 1990 Eurocode 0: Basis of structural design, 2006.

[17] Vincenzo Franciosi. Fondamenti di scienza delle costruzioni, volume II - Teoria della trave, chapter IX. Liguori, 1978.

[18] A. Paglietti and G. Carta. La favola del taglio efficace nella teoria delle travi di altezza variabile. In AIMETA, 2007.

[19] A. Paglietti and G. Carta. Remarks on the current theory of shear strength of variable depth beams. The open civil engineering journal, 3 : $28-33,2009$.

[20] Dewey H Hodges, Jimmy C Ho, and Wenbin Yu. The effect of taper on section constants for in-plane deformation of an isotropic strip. Journal of Mechanics of Materials and Structures, 3(3):425-440, 2008.

[21] S. Timoshenko and D.H. Young. Theory of Structures [by] S.P. Timoshenko [and] D.H. Young. McGraw-Hill, 1965.

[22] Ferdinando Auricchio, Giuseppe Balduzzi, and Carlo Lovadina. A new modeling approach for planar beams: finite-element solutions based on mixed variational derivations. Journal of Mechanics of Materials and Structures, 5(5):771-794, 2010.

[23] G. Balduzzi. Beam Models: Variational Derivation, Analytical and Numerical Solutions. PhD thesis, Universitá degli Studi di Pavia, 2013.

[24] O.A. Bauchau and J.I. Craig. Structural Analysis: With Applications to Aerospace Structures. Solid Mechanics and Its Applications. Springer, 2009.

[25] J.N. Reddy. Energy Principles and Variational Methods in Applied Mechanics. Wiley, 2002.

[26] Stephen M. Alessandrini, Douglas N. Arnold, Richard S. Falk, and Alexandre L. Madureira. Derivation and justification of plate models by variational methods. CRM Proceedings and Lecture Notes, 21:1-20, 1999.

[27] Franco Brezzi and Klaus-Jürgen Bathe. A discourse on the stability conditions for mixed finite element formulations. Computer Methods in Applied Mechanics and Engineering, 82:27-57, 1990.

[28] D. Boffi, F. Brezzi, and M. Fortin. Mixed Finite Element Methods and Applications. Springer Series in Computational Mathematics. Springer, 2013.

[29] Douglas Arnold, Gerard Awanou, and Ragnar Winther. Finite elements for symmetric tensors in theree dimensions. Mathematics of Computation, 263:1229-1251, 2008.

[30] R. Hazrat. Mathematica $($ : A Problem-Centered Approach. Springer Undergraduate Mathematics Series. Springer, 2010.

[31] ABAQUS User's and theory manuals - Release 6.11. Simulia, Providence, RI, USA., 2011.

[32] Otto T.Bruhns. Advanced Mechanics of Solids. Springer, 2003. 